\title{
Micro-osteoperforations (Minimally Invasive Corticotomy Procedure for Accelerated Orthodontic Treatment): A Case Report
}

\author{
Megha Nichal ${ }^{1}$, Vidya Dodwad ${ }^{2}$, Sachin Mangalekar ${ }^{3}$, Priyanka Vhanmane ${ }^{4}$, Sandeep Patil ${ }^{5}$, Smruti J Lulla ${ }^{6}$
}

\begin{abstract}
Micro-osteoperforation is an effective, minimally invasive procedure to accelerate tooth movement and significantly reduces the duration of orthodontic treatment. This technique has several advantages including faster tooth movement, enhanced post-orthodontic treatment stability. In this case report, an adult patient undergoing orthodontic treatment was referred to the department of periodontology for the opinion regarding corticotomy-assisted orthodontic treatment. A full thickness mucoperiosteal flap was reflected on buccal side, micro-osteoperforations were made using a round bur with a micro-motor and only 3 micro-osteoperforations were made to reduce the invasiveness. This approach assumed minimum trauma to the cortical bone. Pre- and postoperative measurements were done on study casts on the day of surgery and after 45 days. Micro-osteoperforation site had a space closure of $1.5 \mathrm{~mm}$ compared to the control site. Micro-osteoperforations significantly increased the rate of tooth movement by 1-2-fold. The patient did not report any significant discomfort during or after the procedure.
\end{abstract}

Keywords: Canine retraction, Corticotomy, Microosteoperforations.

Journal of Health Sciences \& Research (2019): 10.5005/jp-journals-10042-1072

\section{INTRODUCTION}

Recently there has been an increased tendency for researchers to focus on methods accelerating orthodontic tooth movement. A steady rise is seen in the number of adolescent and adult patients seeking orthodontic treatment due to increased awareness amongst people regarding dental and facial esthetics. Adults have more specific objectives and concerns related to dentofacial esthetics, the type of orthodontic appliance and the duration of treatment. ${ }^{1}$ Long treatment time is of prime concern which makes patients decline orthodontic treatment and opt for alternative treatments such as removable partial denture, fixed partial denture or veneers with less than optimum results.

Various new approaches to shorten the treatment time without foregoing optimal results have been introduced till date. These attempts mainly fall into 3 categories. ${ }^{3}$ The first is local or systemic administration of medicines such as prostaglandins, interleukins, leukotrienes, cyclic adenosine monophosphate and vitamin $\mathrm{D}$. These drugs can accelerate periodontal regeneration by inducing orthodontic inflammation, facilitating the formation of osteoclasts from precursor monocytes or improving capillary permeability. ${ }^{2}$ However, the effects of such drugs are not specific and their side effects in the long term are unknown. The second category is mechanical or physical stimulation which includes use of direct electrical current, pulsed electromagnetic field, samarium cobalt magnet and photobiomodulation by laser irradiation. Such methods also have certain drawbacks like lack of patient's compliance, being expensive etc. The last category includes surgical techniques like corticotomy, dento-alveolar or periodontal distraction, and osteotomy that are routinely used for accelerating tooth movement. ${ }^{3}$

Surgically assisted orthodontic tooth movement has been used since the 1800s. Corticotomy-facilitated tooth movement was first described by Bryan in 1893 published in the textbook by

\footnotetext{
${ }^{1-6}$ Department of Periodontology, Bharati Vidyapeeth Dental College, Sangli, Maharashtra, India
}

Corresponding Author: Sachin Mangalekar, Department of Periodontology, Bharati Vidyapeeth Dental College, Sangli, Maharashtra, India, Phone: +91 8871199966, e-mail: sachinbm@ yahoo.com

How to cite this article: Nichal M, Dodwad V, Mangalekar S, et al. Micro-osteoperforations (Minimally Invasive Corticotomy Procedure for Accelerated Orthodontic Treatment): A Case Report. J Health Sci Res 2019;10(1):18-21.

Source of support: Nil

Conflict of interest: None

Guiliford. It was Henrich Kole's publication in 1959, however, that set the stage for evolution of corticotomy-facilitated orthodontics. ${ }^{4}$ Reintroduced in the late 20th century, corticotomy has aroused much curiosity and controversy, fueled, in part, at least by the promotional and commercial interest of the professionals who put it back into the orthodontic scenery. ${ }^{5}$ Corticotomy is the method in which intentional cutting, perforation or mechanical alteration of only cortical bone is done leaving intact the medullary vessels and endosteum inducing regional acceleratory phenomenon (RAP). ${ }^{6}$ Regional acceleratory phenomenon is a local response to a noxious stimulus, which describes a process by which tissue forms faster than the normal regional regeneration process. Simply stated, when bone is surgically irritated, wound is created. This wound initiates a localized inflammatory response. Due to the presence of the inflammatory markers, osteoclasts migrate to the area and cause bone resorption. This effect, however, is temporary and lasts for about 4-6 months. ${ }^{6}$

On the basis of RAP several corticotomy procedures were introduced, out of which a new lesser invasive technique called

(c) The Author(s). 2019Open Access This article is distributed under the terms of the Creative Commons Attribution 4.0 International License (https://creativecommons. org/licenses/by-nc/4.0/), which permits unrestricted use, distribution, and non-commercial reproduction in any medium, provided you give appropriate credit to the original author(s) and the source, provide a link to the Creative Commons license, and indicate if changes were made. The Creative Commons Public Domain Dedication waiver (http://creativecommons.org/publicdomain/zero/1.0/) applies to the data made available in this article, unless otherwise stated. 
micro-osteoperforation (MOP) was introduced by Alikhani et al. This technique involves surgical raising of flap exposing the alveolar bone and making of three MOPs distal to canine, at the level of root apex, $2 \mathrm{~mm}$ above alveolar ridge and at the center of these two perforations. ${ }^{7,8}$

Taking this into consideration, a case was performed with MOPs, and the rate of canine retraction was measured and compared with conventional method using study models.

\section{Case Description}

A 36-year-old female patient was referred to the department of periodontics of the BVDU Dental College and Hospital, Sangli, Maharashtra, India. The patient was concerned about her dentofacial esthetics and demanded shorter duration of treatment time. She had a class 2 div 1 malocclusion, and extraction of all first premolars were done (Fig. 1A). Patient was systemically healthy and with periodontally healthy condition.

\section{Surgical Procedure}

Alginate impressions for study models were taken before canine retraction and on 45th day of canine retraction. The patient received MOPs on the right side as described by Alikhani et al. (2013). The left side acted as (Fig. 1B) the control side which received no MOPs. Full thickness flap was raised (Fig. 1C) and three MOPs were performed (right side) under local anesthesia (2\% lidocaine with 1:100,000 epinephrine) distal to the canines at the level of canine root apex, $2 \mathrm{~mm}$ above alveolar ridge and at the center of these two perforations using a micro-motor (Fig. 1D). Using a round bur, perforations of around $1.5 \mathrm{~mm}$ wide and 2-3 $\mathrm{mm}$ deep were performed. After MOPs, the flap was sutured back to its original position using a braided black silk suture (Fig. 1E) and patient was put on analgesics three times a day for five days. Followed by MOPs immediate orthodontic force of $200 \mathrm{mg}$ was applied for canine retraction on both control and on study side using NiTi closed coil spring connected from molar hook to canine bracket hook. Force level was checked using a Dontrix force gauge by the orthodontist. At each visit, the appliances were monitored for any deformation or change in position because of chewing. Second set of maxillary alginate impressions records were taken on 45th day after canine retraction of each patient to monitor the rate of tooth movement. Vertical lines were drawn on the study model over the palatal surface of the canine from the middle of the cuspal tip to the middle of the cervical line and middle of incisal edge to middle of cervical line of lateral incisor (Fig. 2A). The distance between the long axis of canine and the lateral incisor was assessed before and after canine retraction at 3 levels at incisal (D1), middle (D2) and cervical thirds (D3) of the crowns as shown in the Figure 2B. All measurements on the study models were done using digital caliper (Fig. 2C). The amount of canine retraction was evaluated on 45th day using a digital caliper (Table 1).

From the above readings there was a space closure of $1.5 \mathrm{~mm}$ at the MOP site whereas in the control site the space closure was up to $0.29 \mathrm{~mm}$. Canine retraction was found to be faster in the MOP site.

The figure below shows space closure at the control and MOP site (A) before canine retraction and (B) on the 45th day of canine retraction (Fig. 3).

\section{Discussion}

Periodontic orthodontic interactions are mutually beneficial. The combined approach can greatly enhance the periodontal health and dentofacial esthetics in many situations. It is the result of sincere effort, intelligent direction, skillful execution and the vision to see obstacles as opportunities. Given this constant demand for shorter treatment time from around the world, researchers have increasingly sought various ways to boost orthodontic treatment efficiency. Assuming that mechanotherapy and cooperation are optimized for any given patient, the rate-limiting step in treatment time will be the patient's biological response to mechanotherapy. Thus, identifying and harnessing the cellular regulators of tooth movement are essential if we are to safely shorten orthodontic treatment time.
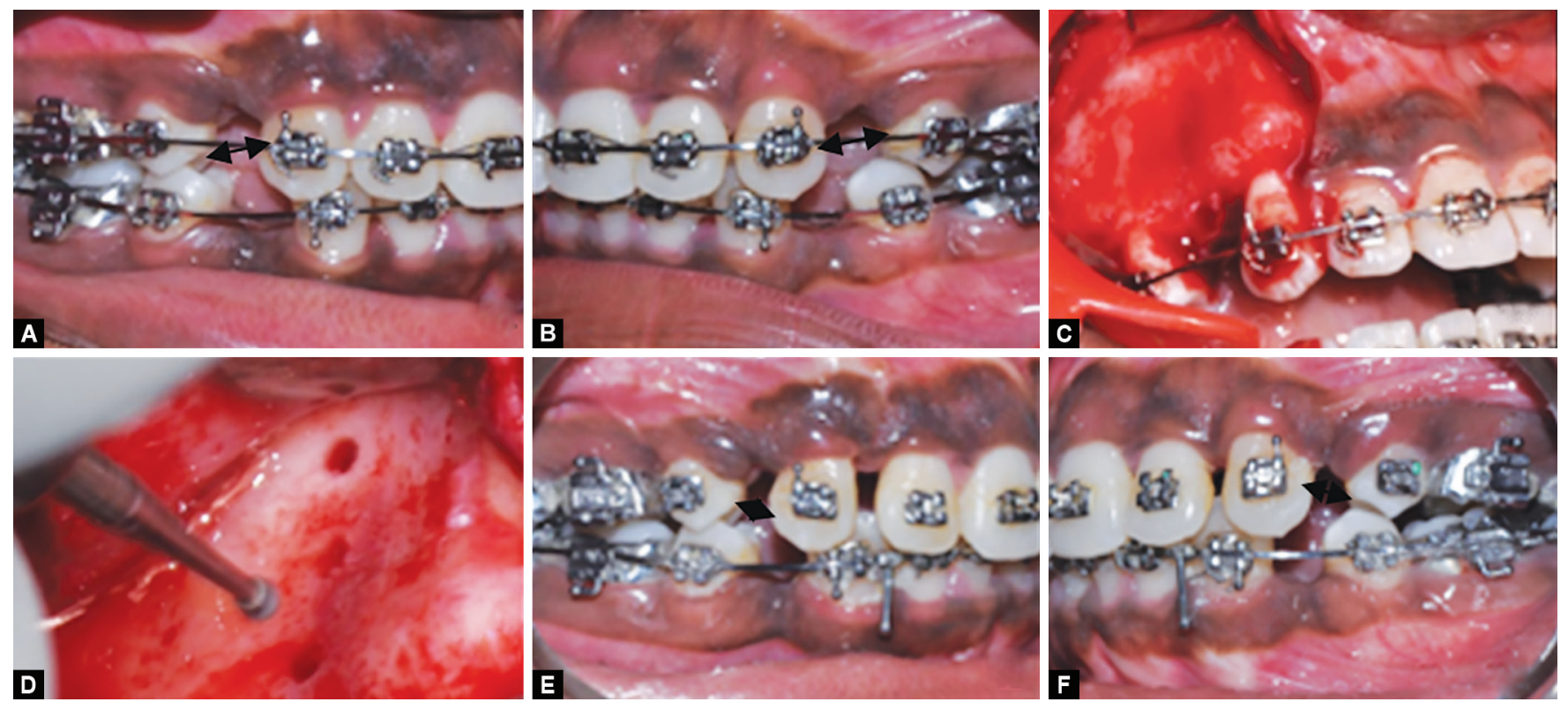

Figs 1 A to F: Surgical procedure; (A) Preoperative right lateral view; (B) Preoperative left lateral view; (C) Full thickness mucoperiosteal flap reflected; (D) Micro-osteoperforation (MOP) using a round bur; (E) Postoperative 45 days MOP site; (F) Postoperative 45 days control site 

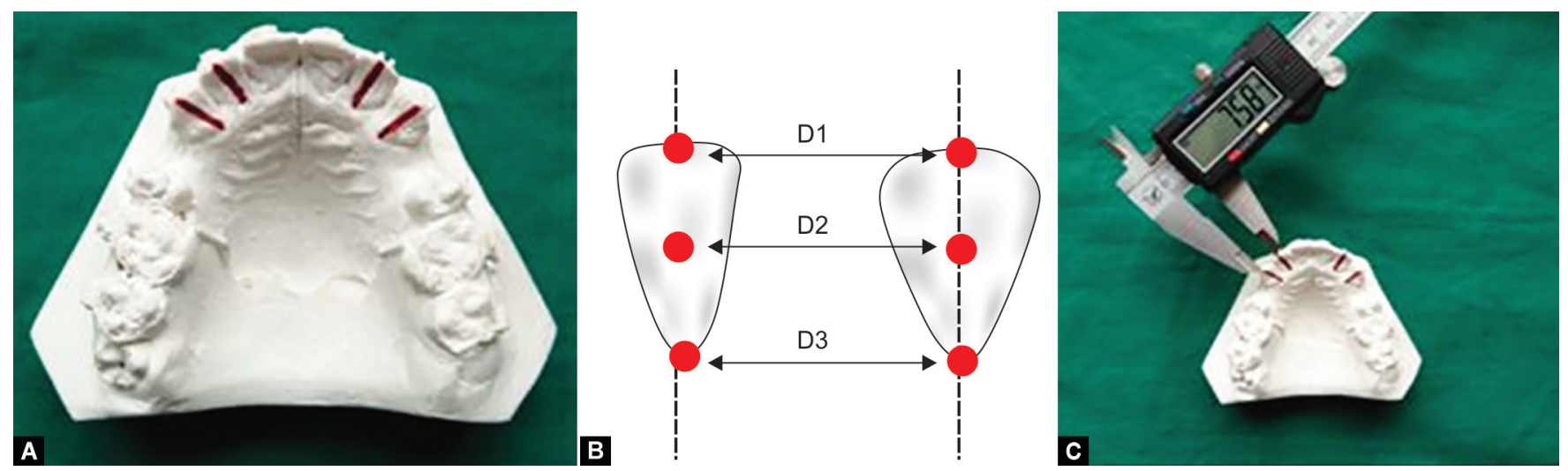

Figs 2 A to C: Method of measuring using a digital caliper; (A) Long axis marked on lateral incisor and canine; (B) (D1) Distance measured between long axis of lateral incisor and canine at the incisal level. (D2) Distance measured between long axis of lateral incisor and canine at the middle level. (D3) Distance measured between the long axis of the lateral incisor and canine at the cervical level; (C) Measurement using a digital caliper

Table 1: Pre- and postoperative readings at the control and MOP sites

\begin{tabular}{lllll}
\hline \multirow{2}{*}{ Control } & Pre & D1 & D2 & D3 \\
& Post & 9.04 & 7.02 & 5.5 \\
\multirow{3}{*}{ MOP } & 9.2 & 7.34 & 5.9 \\
& Pre & 8.01 & 7.58 & 6.52 \\
& Post & 9.75 & 9.51 & 7.46 \\
\hline
\end{tabular}

Exploring the surgical methods which reduce the treatment duration without compromising the treatment outcome have been challenging. According to Long et al. corticotomy has been proved to be very efficient, safe and time saving. A systemic review by Long et al. ${ }^{9}$ concluded that amongst various methods used for accelerating orthodontic tooth movements, corticotomy surgical method was a safe, efficient and timereducing procedure.

Generally, the conventional corticotomy techniques include both labial and lingual cuts and sometimes require two-stage surgery. Although the clinical healing was uneventful in these conventional techniques, some complications such as subcutaneous hematomas of the face and the neck, pain, swelling, root resorption and the possibility of infection could occur after intensive corticotomies. ${ }^{1,8,10,11}$ Hence, to overcome all these disadvantages, many modified techniques of corticotomy have evolved.

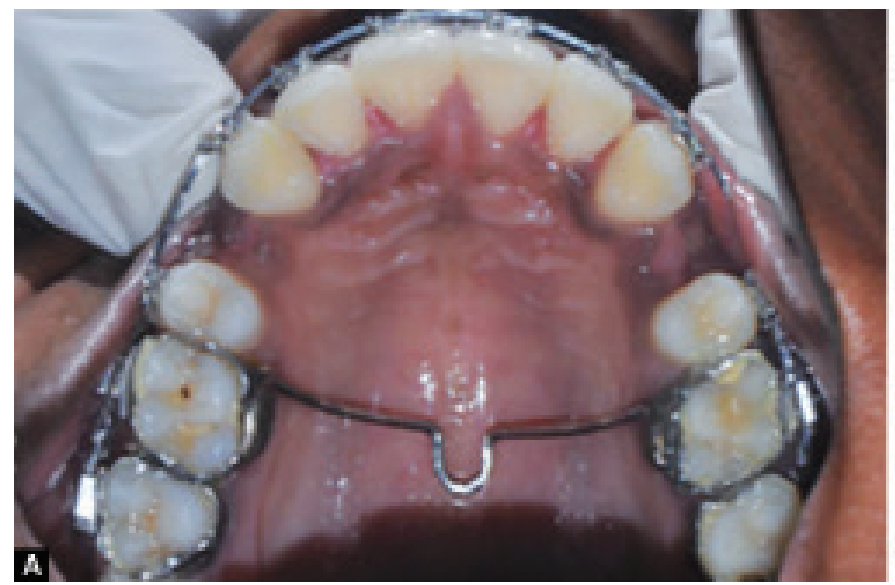

Germec $^{12}$ proposed modified corticotomy technique, in which the lingual vertical and subapical horizontal cuts were eliminated. He concluded that corticotomy-facilitated orthodontics dramatically reduced the treatment time without any adverse effects on the periodontium and the vitality of the teeth. The main advantages of this modified corticotomy technique were the elimination of the lingual cuts and flap, time-saving procedure and minimum discomfort to the patient.

A more recent surgical orthodontic therapy was introduced by Wilcko et al. 5,10,14 which included the innovative strategy of combining corticotomy surgery with alveolar grafting. This technique reduces treatment time to one-third the time of conventional orthodontics. ${ }^{13}$ Several reports indicated that this technique is safe, effective, extremely predictable, associated with less root resorption and reduced treatment time, also can reduce the need for orthognathic surgery in certain situations. But limitations of this technique are its high cost, technique sensitivity and patient's compliance.

Aboul-Ela ${ }^{15}$ has done a study in which they did modified corticotomy with reflection of buccal flap. Micro-osteoperforations were performed using a round bur in a slow speed hand piece on one side and on the other side conventional method for retraction of canine was used. Perforations were made extending from the lateral incisor to first premolar area. In this study, they found that the experimental side showed a faster rate of retraction. From this study, they concluded that, corticotomy helps in increasing the tooth

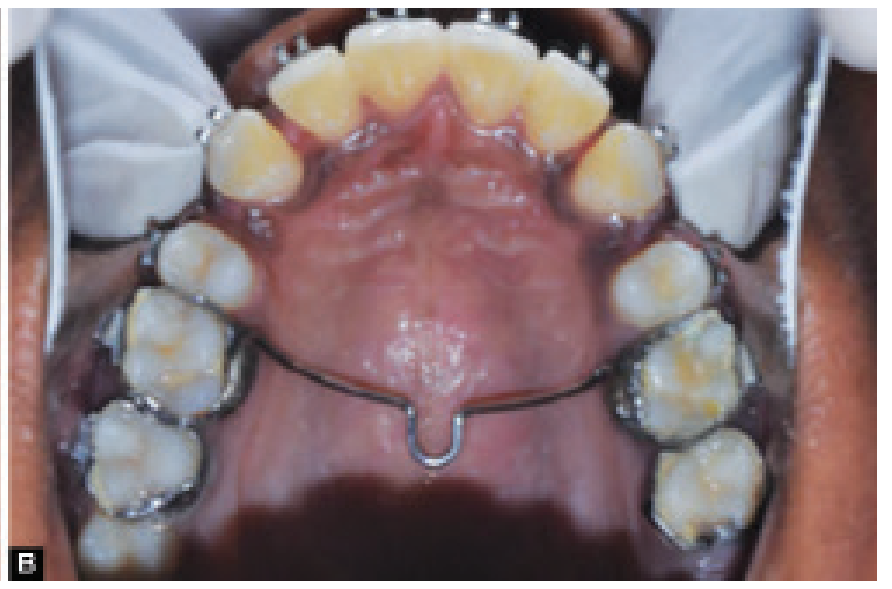

Figs 3A and B: (A) Before canine retraction; (B) 45th day of canine retraction 
movement without any ill effects to the patient. ${ }^{16}$ This result agrees with the findings of Wilcko et al., suggesting that the rapid rate of tooth movement seems to depend mostly on RAP.

To reduce the invasive nature of surgical irritation of bone, a device called Propel was introduced by Propel Orthodontics. They termed this process as alveocentesis which literally translates to puncturing bone. The use of this device in animals has shown that performing MOPs on alveolar bone during orthodontic tooth movement can stimulate the expression of inflammatory markers which leads to increase in osteoclast activity and rate of tooth movement. Alikhani et al. ${ }^{7}$ performed a single center single blinded study to investigate this procedure on humans. Only three MOPs were made distal to canine. He found that MOPs significantly increased the expression of cytokines and chemokines which are known to recruit osteoclast precursors and stimulate osteoclast differentiation. MOPs increased the rate of canine retraction 2.3-fold compared to the control group. However, this technique is quite expensive as they have used Propel for performing micro-osteoperforations.

Considering the advantages of techniques given by Alikhani et al. ${ }^{7}$ and Aboul-Ela we did 2 cases with the aim of evaluating the amount of canine retraction following micro-osteoperforations using study models as the measuring tool.

In this case report we had reflected full thickness mucoperiosteal flap on the buccal side and MOPs were made using a round bur with micro-motor as described by Aboul-Ela and only three MOPs were made to reduce the invasiveness as tried by Alikhani et al. ${ }^{7}$ This approach assumed minimum trauma to the cortical bone.

Study models were used to measure the amount of canine retraction as they are more patient friendly, cost effective and changes can be easily appreciated.

In this case, no postoperative complications were observed. The advantages of this technique over conventional techniques are that it is a minimally invasive procedure (only three MOPs), involves undermining of cortical bone only, and is a cost effective and time saving procedure.

The result of this case report showed that mean movement in MOP site was greater when compared to control site on study models which is in accordance with the study by Aboul-Ela. The average daily rate of canine retraction was significantly higher on the corticotomy than the control side by 2 times during the first 2 months after the corticotomy surgery. This rate of tooth movement declined to only 1.1 times higher in the third month and 1.06 times higher by the end of the fourth month. But in this study the number of perforations were more when compared our case report. But the results in both were similar showing a greater amount of canine retraction on the microosteoperforations side.

A thorough case analysis should be done while selecting cases for MOP. The surrounding anatomical limitations, such as maxillary sinus pneumatization or narrowed alveolar ridge should be considered. One of the obvious obstacles in the surgical procedure is the maxillary sinus pneumatization which is an anatomical limitation of bone reduction in depth. Caution must be taken to avoid mechanical trauma to the root of the tooth during the surgical procedure as this approach is technique-sensitive as mentioned earlier.

\section{Conclusion}

The present case report has shown the effects of microosteoperforations on accelerating the tooth movement. It significantly increases the expression of inflammatory markers which recruit osteoclast precursors and stimulate osteoclast differentiation. MOP is a minimal invasive method which significantly increases the rate of canine retraction and can reduce orthodontic treatment time.

Despite the increased rate of canine movement, it must be kept in mind that the long-term effects of this technique on pulpal vitality, root resorption and periodontal tissue has to be evaluated on broad basis. However, further studies on the effect of the number of MOPs on the rate of tooth movement should be carried out.

\section{References}

1. Amit Goyal, Kalra JPS, et al. Periodontally accelerated osteogenic orthodontics (PAOO)- A review. J Clin Exp Dent 2012;4(5):292-296. DOI: $10.4317 /$ jced.50822.

2. Krishnana V, Davidovitch Z. Celluar, molecular and tissue levelreactions to orthodontic force. Am J Orthod Dentofacial Orthop 2006;129(4):469.e1-469.e32. DOI: 10.1016/j.ajodo.2005.10.007.

3. Ren A, LvT, et al. Rapid orthodontic tooth movement aided by alveolar surgery in beagles. Am J Orthod Dentofacial Orthop 2007;131(2):160. e1-160.e10. DOI: 10.1016/j.ajodo.2006.05.029.

4. Karanth S, Ramesh A, et al. Periodontallyaccelerated orthodontics: Review on a surgical technique and case report. J Interdiscip Dent 2012;2(3):179-184. DOI: 10.4103/2229-5194.113250.

5. Murphy KG, Wilko MT, et al. Periodontal Accelerated Osteogenic Orthodontics: A Description of the Surgical Technique.J Oral Maxillofac Surg 2009;67(1):2160-2166. DOI: 10.1016/j.joms.2009.04.124.

6. Sebaoun JD, Kantarci A, et al. Modeling of trabecular bone and lamina dura following selective alveolardecortication in rats. J Periodontol 2008;79(9):1679-1688. DOI: 10.1902/jop.2008.080024.

7. Alikhani M. Effect of micro-osteoperforations on the rate of tooth movement. Am J Orthod Dentofacial Orthop 2013;144(5):639-648. DOI: 10.1016/j.ajodo.2013.06.017.

8. John Wiley and Sons. Wiley Blackwell Orthodontically Driven Corticotomy-Text book. Burgnami F, Calazzo A ed.; 2015.

9. Long $\mathrm{H}$, Pyakurel $\mathrm{U}$, et al. Interventions foraccelerating orthodontic tooth movement: a systematic review. Angle Orthod 2013;83(1): 164-171. DOI: 10.2319/031512-224.1.

10. Wilcko MT, Wilcko WM, et al. Accelerated Osteogenic Orthodontics Technique: A 1-StageSurgically Facilitate Rapid Orthodontic Technique With Alveolar Augmentation. A J Oral Maxillofac Surg 2009;67:2149-2159. DOI: 10.1016/j.joms.2009.04.095.

11. Thafeed AS, Al Ghamdi. Corticotomy facilitated orthodontics: Review of a technique. The Saudi Dental Journal 2009;4(1):159-164.

12. Germec D, Giray B, et al. Lower incisor retraction with amodified corticotomy. Angle Orthod 2006;76(5):882-890. DOI: 10.1043/ 0003-3219(2006)076[0882:LIRWAM]2.0.CO;2.

13. Sirisha K, Srinivas M, et al. Pratap Gowd Wilkodontics- A Novel Synergy in Time to Save Time. J Clin Diagn Res 2014;8(1):322-325. DOI: 10.7860/JCDR/2014/7576.3978.

14. Wilcko WM, Wilcko MT, et al. Accelerated Orthodontics with alveloar reshaping. J Ortho Practice 2000;10(1):63-70.

15. Yang C, Wang C, et al. Biomechanical effects of corticotomyapproaches on dentoalveolar structures during canine retraction: a 3-dimensionalfinite element analysis. Am J Orthod Dentofacial Orthop 2015;148(3):457-465. DOI: 10.1016/j.ajodo.2015.03.032.

16. Gadakh SB, Gulve N, et al. Methods of Accelerating orthodontic treatment - A Review. J appl dent Med Sci 2016;2(1):176-182. 